International Journal of Pure and Applied Mathematics

Volume 83 No. $3 \quad 2013,405-416$

ISSN: 1311-8080 (printed version); ISSN: 1314-3395 (on-line version)

url: http://www.ijpam.eu

doi: http://dx.doi.org/10.12732/ijpam.v83i3.3

\title{
CONTINUOUS BLOCK METHOD FOR THE SOLUTION OF SECOND ORDER INITIAL VALUE PROBLEMS OF ORDINARY DIFFERENTIAL EQUATION
}

\author{
A. Adewale James ${ }^{1 \S}$, A. Olaide Adesanya ${ }^{2}$, Sunday Joshua ${ }^{3}$ \\ ${ }^{1}$ Mathematics Division \\ American University of Nigeria \\ Yola, Adamawa State, NIGERIA \\ ${ }^{2}$ Department of Mathematics \\ ModibboAdama University of Technology \\ Yola, Adamawa State, NIGERIA \\ ${ }^{3}$ Department of Mathematical Sciences \\ Adamawa State University \\ Mubi, Adamawa State, NIGERIA
}

\begin{abstract}
We proposed a continuous blocks method for the solution of second order initial value problems with constant step size in this paper. The method was developed by interpolation and collocation of power series approximate solution to generate a continuous linear multistep method;this is evaluated for the independent solution to give a continuous block method which is evaluated at selected grid point to give discrete block method. The basic properties of the method were investigated and was found to be zero stable, consistent and convergent. The efficiency of the method was tested on some numerical examples and found to give better approximation than the existing methods.
\end{abstract}

AMS Subject Classification: 65205, 65L06, 65D30

Key Words: collocation, continuous block method, interpolation, approximate solution

Received: September 24, 2012

(C) 2013 Academic Publications, Ltd. url: www.acadpubl.eu

${ }^{\S}$ Correspondence author 


\section{Introduction}

This paper considers a numerical solution to the second order Initial value problems of the form

$$
y^{\prime \prime}=f\left(x, y, y^{\prime}\right), \quad y\left(x_{0}\right)=y_{0}, \quad y^{\prime}\left(x_{0}\right)=y_{0}^{\prime},
$$

where $x_{0}$ is the initial point and $f$ is continuous within the internal of integration.

Scholars have established that direct methods of solving higher order ordinary differential equations are better than the method of reduction to systems of first order ordinary differential equation (Adesanya et al [2], Awoyemi [6]), hence the study of direct method for the solution of higher order ordinary differential equation is important.

Adoption of method of collocation and interpolation of power series approximate solution to developed an implicit continuous linear multistep method have been studied by many scholars, among them are Fatunla [8], Awoyemi and Kayode [7], Olabode [14], Adesanya et al. [4], Kayode and Awoyemi [13]. These authors independently implemented their methods in predictor corrector mode, where the predictor are reducing order predictor hence this affect the accuracy of the method. Other setbacks of these method as extensively discussed by Adesanya et al. [3] and Awoyemi [6].

In order to cater for the shortcoming of the predictor-corrector method, scholars adopted block method which is self-starting and does not need starting value to be implemented. Among these authors are: Jator [12], Jator and $\mathrm{Li}$ [11], Omar and Suleiman ([15], Abass [1], Fudziah, Yab and Mohammed [9], Awoyemi et al. [5].

In this paper, we propose continuous block method which has the same properties as the continuous linear multistep method. This continuity properties enable us to evaluate at all points within the interval of integration, hence enable the study of the dynamical system at all the grid points. This paper considers four off step points which makes it an improvement on the work of Jator [12] which considered two off step points.

\section{Methodology}

We first state the theorem that establishes the uniqueness of solutions of higher order ordinary differential equations. 
Theorem 1. Let

$$
U^{(n)}=f\left(x, u, u^{\prime}, \ldots, u^{(n-1)}\right), U^{k}\left(x_{0}\right)=C_{k}
$$

$k=0,1, \ldots,(n-1), u$ and $f$ are scalars. Let $R$ be the region defined by the inequalities $x_{0} \leq x \leq x_{0}+a, \quad\left|s_{j}-c_{j}\right| \leq b, j=0,1, \ldots, n-1(a>0, b>0)$. Suppose the function $f\left(x, s_{0}, s_{1}, \ldots, s_{n-1}\right)$ is defined in $R$ and in addition:

(a) $f$ is non-negative and non-decreasing in each $x, s_{0}, s_{1}, \ldots, s_{n-1}$ in $R$;

(b) $f\left(x, c_{0}, c_{1}, \ldots, c_{n-1}\right)>0$ for $x_{0} \leq x \leq x_{0}+a$; and

(c) $C_{k} \geq 0, \quad k=0,1,2, \ldots, n-1$.

Then value problem (2) has a unique solution in $R$ (See Wend (1967) for details).

\subsection{Specification of the Method}

We considered a power series approximate solution of the form

$$
y(x)=\sum_{j=0}^{r+s-1} a_{j} x^{j},
$$

where $r$ and $s$ are the numbers of interpolation and collocation points respectively. The second derivatives of (2) gives

$$
y^{\prime \prime}(x)=\sum_{j=2}^{r+s-1} j(j-1) a_{j} x^{j-2} .
$$

Substituting (3) into (1) gives

$$
f\left(x, y, y^{\prime}\right)=\sum_{j=2}^{r+s-1} j(j-1) a_{j} x^{j-2} .
$$

Let the solution to (1) be soughted on the partition $N: a=x_{0}<x_{1}<x_{2}<$ $\ldots<x_{N}=b$ with a constant step size $(h)$ given as $h=x_{n+1}-x_{n}, n=0,1, \ldots, N$

Collocating (4) at $x_{n+r}, r=0\left(\frac{1}{3}\right) 2$ and interpolating (2) at $x_{n+s}, s=0,1$ gives a system of non linear equation of the form

$$
A X=U,
$$

where

$$
A=\left[\begin{array}{lllllllll}
a_{0} & a_{1} & a_{2} & a_{3} & a_{4} & a_{5} & a_{6} & a_{7} & a_{8}
\end{array}\right]^{T},
$$




$$
U=\left[\begin{array}{lllllllll}
y_{n} & y_{n+1} & f_{n} & f_{n+\frac{1}{3}} & f_{n+\frac{2}{3}} & f_{n+1} & f_{n+\frac{4}{3}} & f_{n+\frac{5}{3}} & f_{n+2}
\end{array}\right]^{T},
$$

and

$$
\begin{aligned}
& X= \\
& {\left[\begin{array}{lllllllll}
1 & x_{n} & x_{n}^{2} & x_{n}^{3} & x_{n}^{4} & x_{n}^{5} & x_{n}^{6} & x_{n}^{7} & x_{n}^{8} \\
1 & x_{n+1} & x_{n+1}^{2} & x_{n+1}^{3} & x_{n+1}^{4} & x_{n+1}^{5} & x_{n+1}^{6} & x_{n+1}^{7} & x_{n+1}^{8} \\
0 & 0 & 2 & 6 x_{n} & 12 x_{n}^{2} & 20 x_{n}^{3} & 30 x_{n}^{4} & 42 x_{n}^{5} & 56 x_{n}^{6} \\
0 & 0 & 2 & 6 x_{n+\frac{1}{3}} & 12 x_{n+\frac{1}{3}}^{2} & 20 x_{n+\frac{1}{3}}^{3} & 30 x_{n+\frac{1}{3}}^{4} & 42 x_{n+\frac{1}{3}}^{5} & 56 x_{n+\frac{1}{3}}^{6} \\
0 & 0 & 2 & 6 x_{n+\frac{2}{3}} & 12 x_{n+\frac{2}{3}}^{2} & 20 x_{n+\frac{2}{3}}^{3} & 30 x_{n+\frac{2}{3}}^{4} & 42 x_{n+\frac{2}{3}}^{5} & 56 x_{n+\frac{2}{3}}^{6} \\
0 & 0 & 2 & 6 x_{n+1} & 12 x_{n+1}^{2} & 20 x_{n+1}^{3} & 30 x_{n+1}^{4} & 42 x_{n+1}^{5} & 56 x_{n+1}^{6} \\
0 & 0 & 2 & 6 x_{n+\frac{4}{3}} & 12 x_{n+\frac{4}{3}}^{2} & 20 x_{n+\frac{4}{3}}^{3} & 30 x_{n+\frac{4}{3}}^{4} & 42 x_{n+\frac{4}{3}}^{5} & 56 x_{n+\frac{4}{3}}^{6} \\
0 & 0 & 2 & 6 x_{n+\frac{5}{3}} & 12 x_{n+\frac{5}{3}}^{2} & 20 x_{n+\frac{5}{3}}^{3} & 30 x_{n+\frac{5}{3}}^{4} & 42 x_{n+\frac{5}{3}}^{5} & 56 x_{n+\frac{5}{3}}^{6} \\
0 & 0 & 2 & 6 x_{n+2} & 12 x_{n+2}^{2} & 20 x_{n+2}^{3} & 30 x_{n+2}^{4} & 42 x_{n+2}^{5} & 56 x_{n+2}^{6}
\end{array}\right] .}
\end{aligned}
$$

Solving (5) for $\mathrm{a}_{j}^{\prime} s$ using Guassian elimination method and substituting back into (2) gives a continuous hybrid linear multistep method of the form

$$
y(x)=\sum_{j=0}^{1} \alpha_{j} y_{n+j}+h^{2}\left(\sum_{j=0}^{1} \beta_{j}(x) f_{n+j}+\beta_{k} f_{n+k}\right), k=\frac{1}{3}, \frac{2}{3}, \frac{4}{3}, \frac{5}{3} .
$$

The coefficients of $y_{n+j}, f_{n+j}$ and $f_{n+k}$ give

$$
\begin{gathered}
\alpha_{0}=1-t, \quad \alpha_{1}=t, \\
\beta_{0}=\frac{1}{13440}\left(243 t^{8}-2268 t^{7}+8820 t^{6}-18522 t^{5}+22736 t^{4}-1644 t^{3}\right. \\
\left.+6720 t^{2}-1265 t\right), \\
\beta_{\frac{1}{3}}=-\frac{1}{2240}\left(243 t^{8}-2160 t^{7}+7812 t^{6}-14616 t^{5}+14616 t^{4}-6720 t^{3}+825 t\right), \\
\beta_{\frac{2}{3}}=\frac{1}{4480}\left(1215 t^{8}-10260 t^{7}+34524 t^{6}-58086 t^{5}+49140 t^{4}-16800 t^{3}-267 t\right), \\
\beta_{1}=-\frac{1}{3360}\left(1215 t^{8}-9720 t^{7}+30492 t^{6}-46872 t^{5}+35560 t^{4}-11200 t^{3}+525 t\right), \\
\beta_{\frac{4}{3}}=-\frac{1}{2240}\left(243 t^{8}-1728 t^{7}-4788 t^{6}-6552 t^{5}+4536 t^{4}-1344 t^{3}+57 t\right),
\end{gathered}
$$




$$
\begin{aligned}
& \beta_{\frac{5}{3}}=-\frac{1}{2240}\left(243 t^{8}-1728 t^{7}-4788 t^{6}-6552 t^{5}+4536 t^{4}-1344 t^{3}+57 t\right), \\
& \beta_{2}=\frac{1}{13440}\left(243 t^{8}-1620 t^{7}+4284 t^{6}-5670 t^{5}+3836 t^{4}-1120 t^{3}+47 t\right) .
\end{aligned}
$$

where $t=\frac{x-x_{\mathrm{n}}}{h}, \quad y_{n+j}=y\left(x_{n}+j h\right)$ and

$$
f_{n+j}=f\left(\left(x_{n+j} h\right), y\left(x_{n}+j h\right), y^{\prime}\left(x_{n}+j h\right)\right) .
$$

Solving (6) for the independent solution at the grid points gives the continuous block method

$$
y(x)=\sum_{j=0}^{1} \frac{(j h)^{(m)}}{m !} y_{n}^{(m)}+h^{2}\left(\sum_{j=0}^{1} \sigma_{j}(x) f_{n+j}+\sigma_{k} f_{n+k}\right), k=\frac{1}{3}, \frac{2}{3}, \frac{4}{3}, \frac{5}{3} .
$$

The coefficient of $f_{n+j}$ and $f_{n+k}$ give

$$
\begin{gathered}
\sigma_{0}=\frac{1}{13440}\left(243 t^{8}-2268 t^{7}+8820 t^{6}-18522 t^{5}+22736 t^{4}-1644 t^{3}+6720 t^{2}\right), \\
\beta_{\frac{1}{3}}=-\frac{1}{2240}\left(243 t^{8}-2160 t^{7}+7812 t^{6}-14616 t^{5}+14616 t^{4}-6720 t^{3}\right), \\
\beta_{\frac{2}{3}}=\frac{1}{4480}\left(1215 t^{8}-10260 t^{7}+34524 t^{6}-58086 t^{5}+49140 t^{4}-16800 t^{3}\right), \\
\beta_{1}=-\frac{1}{3360}\left(1215 t^{8}-9720 t^{7}+30492 t^{6}-46872 t^{5}+35560 t^{4}-11200 t^{3}\right), \\
\beta_{\frac{4}{3}}=-\frac{1}{2240}\left(243 t^{8}-1728 t^{7}-4788 t^{6}-6552 t^{5}+4536 t^{4}-1344 t^{3}\right), \\
\beta_{\frac{5}{3}}=-\frac{1}{2240}\left(243 t^{8}-1728 t^{7}-4788 t^{6}-6552 t^{5}+4536 t^{4}-1344 t^{3}\right), \\
\beta_{2}=\frac{1}{13440}\left(243 t^{8}-1620 t^{7}+4284 t^{6}-5670 t^{5}+3836 t^{4}-1120 t^{3}\right) .
\end{gathered}
$$

Evaluating (7) at $t=\frac{1}{3}\left(\frac{1}{3}\right) 2$ gives a discrete block formula of the form

$$
\mathbf{A}^{(0)} \mathbf{Y}_{m}^{(i)}=\sum_{i=0}^{1} h^{i} \mathbf{e}_{i} y_{n}^{(i)}+h^{2} \mathbf{d}_{\mathbf{i}} f\left(y_{n}\right)+h^{2} \mathbf{b}_{\mathbf{i}} \mathbf{f}\left(\mathbf{Y}_{m}\right), i=0,1
$$

where

$$
\mathbf{Y}_{m}=\left[\begin{array}{llllll}
y_{n+\frac{1}{3}} & y_{n+\frac{2}{3}} & y_{n+1} & y_{n+\frac{4}{3}} & y_{n+\frac{5}{3}} & y_{n+2}
\end{array}\right]^{T}
$$




$$
\begin{gathered}
\mathbf{f}\left(\mathbf{Y}_{m}\right)=\left[\begin{array}{llllll}
f_{n+\frac{1}{3}} & f_{n+\frac{2}{3}} & f_{n+1} & f_{n+\frac{4}{3}} & y_{n+\frac{5}{3}} & y_{n+2}
\end{array}\right]^{T}, \\
\mathbf{y}_{n}^{(i)}=\left[\begin{array}{llllll}
y_{n-1}^{(i)} & y_{n-2}^{(i)} & y_{n-3}^{(i)} & y_{n-4}^{(i)} & y_{n-5}^{(i)} & y_{n}^{(i)}
\end{array}\right]^{T}, \\
\mathbf{f}\left(\mathbf{y}_{n}\right)=\left[\begin{array}{llllll}
f_{n-1} & f_{n-2} & f_{n-3} & f_{n-4} & f_{n-5} & f_{n}
\end{array}\right]^{T},
\end{gathered}
$$

and $A^{(0)}=6 \times 6$ Identity matrix.

If $i=0$, then:

$$
\begin{gathered}
\mathbf{e}_{0}=\left[\begin{array}{cccccc}
0 & 0 & 0 & 0 & 0 & 1 \\
0 & 0 & 0 & 0 & 0 & 1 \\
0 & 0 & 0 & 0 & 0 & 1 \\
0 & 0 & 0 & 0 & 0 & 1 \\
0 & 0 & 0 & 0 & 0 & 1 \\
0 & 0 & 0 & 0 & 0 & 1
\end{array}\right], \quad \mathbf{e}_{1}=\left[\begin{array}{llllll}
0 & 0 & 0 & 0 & 0 & \frac{1}{3} \\
0 & 0 & 0 & 0 & 0 & \frac{2}{3} \\
0 & 0 & 0 & 0 & 0 & 1 \\
0 & 0 & 0 & 0 & 0 & \frac{4}{3} \\
0 & 0 & 0 & 0 & 0 & \frac{5}{3} \\
0 & 0 & 0 & 0 & 0 & 2
\end{array}\right], \\
\mathbf{d}_{0}=\left[\begin{array}{llllllll}
0 & 0 & 0 & 0 & 0 & \frac{28549}{1088640} \\
0 & 0 & 0 & 0 & 0 & \frac{1027}{17010} \\
0 & 0 & 0 & 0 & 0 & \frac{253}{2688} \\
0 & 0 & 0 & 0 & 0 & \frac{1088}{8505} \\
0 & 0 & 0 & 0 & 0 & \frac{35225}{217728} \\
0 & 0 & 0 & 0 & 0 & \frac{41}{210} & & \\
\mathbf{b}_{\mathbf{0}}= & \frac{275}{5184} & \frac{-5717}{120960} & \frac{10621}{272160} & \frac{-7703}{362880} & \frac{403}{60480} & \frac{-199}{217728} \\
\frac{194}{945} & \frac{-8}{81} & \frac{788}{8505} & \frac{-97}{1890} & \frac{46}{2835} & \frac{-19}{8505} & \\
\frac{165}{448} & \frac{-267}{4480} & \frac{5}{32} & \frac{-363}{4480} & \frac{57}{2240} & \frac{-47}{13440} \\
\frac{1504}{2835} & \frac{-8}{945} & \frac{2624}{8505} & \frac{-8}{81} & \frac{32}{945} & \frac{-8}{1701} \\
\frac{8375}{12096} & \frac{3125}{72576} & \frac{25625}{54432} & \frac{-625}{24192} & \frac{275}{5184} & \frac{-1375}{217728} \\
\frac{6}{7} & \frac{3}{35} & \frac{68}{105} & \frac{3}{70} & \frac{6}{35} & 0
\end{array}\right]
\end{gathered}
$$

If $i=1$, then:

$$
\mathbf{e}_{1}=\left[\begin{array}{cccccc}
0 & 0 & 0 & 0 & 0 & 1 \\
0 & 0 & 0 & 0 & 0 & 1 \\
0 & 0 & 0 & 0 & 0 & 1 \\
0 & 0 & 0 & 0 & 0 & 1 \\
0 & 0 & 0 & 0 & 0 & 1 \\
0 & 0 & 0 & 0 & 0 & 1
\end{array}\right], \quad \mathbf{d}_{1}=\left[\begin{array}{llllll}
0 & 0 & 0 & 0 & 0 & \frac{19087}{181440} \\
0 & 0 & 0 & 0 & 0 & \frac{1139}{11340} \\
0 & 0 & 0 & 0 & 0 & \frac{137}{1344} \\
0 & 0 & 0 & 0 & 0 & \frac{286}{2835} \\
0 & 0 & 0 & 0 & 0 & \frac{3715}{36288} \\
0 & 0 & 0 & 0 & 0 & \frac{41}{420}
\end{array}\right]
$$




$$
\mathbf{b}_{1}=\left[\begin{array}{llllll}
\frac{2713}{7560} & \frac{-15487}{60480} & \frac{586}{2835} & \frac{-6737}{60480} & \frac{263}{7560} & \frac{-863}{181440} \\
\frac{94}{189} & \frac{11}{3780} & \frac{332}{2835} & \frac{-269}{3780} & \frac{22}{945} & \frac{-37}{11340} \\
\frac{27}{56} & \frac{-387}{2240} & \frac{34}{105} & \frac{-243}{2240} & \frac{9}{280} & \frac{-29}{6720} \\
\frac{464}{945} & \frac{-128}{945} & \frac{1504}{2835} & \frac{-58}{945} & \frac{16}{945} & \frac{-8}{2835} \\
\frac{725}{1512} & \frac{-2125}{12096} & \frac{250}{567} & \frac{-3875}{12096} & \frac{235}{1572} & \frac{-275}{36288} \\
\frac{18}{35} & \frac{9}{140} & \frac{68}{105} & \frac{9}{140} & \frac{18}{35} & \frac{41}{420}
\end{array}\right] .
$$

\section{Analysis of Basic Properties of the Method}

\subsection{Order of the Block}

Let the linear operator $\{y(x): h\}$ associated with the discrete block method (8) be defined as

$$
\{y(x): h\}=\mathbf{A}^{(0)} \mathbf{Y}_{m}^{(i)}-\sum_{i=0}^{1} h^{i} e_{i} y_{n}^{(i)}-h^{2}\left(\mathbf{d} \mathbf{f}\left(y_{n}\right)+\mathbf{b F}\left(Y_{m}\right)\right) .
$$

Expanding (9) in Taylor series and comparing the coefficients of $h$ gives

$$
\begin{aligned}
\{y(x): h\}=C_{0} y(x)+ & C_{1} y^{\prime}(x)+\ldots+C_{p} h^{p} y^{p}(x)+C_{p+1} h^{p+1} y^{p+1}(x) \\
& +C_{p+2} h^{p+2} y^{p+2}(x)+\ldots
\end{aligned}
$$

Definition 2. The linear operator and associated block formula are said to be of order $p$ if $C_{0}=C_{1}=\ldots=C_{p}=C_{p+1}=0 C_{p+2} \neq 0 . C_{p+2}$ is called the error constant and implies that the truncation error is given by $t_{n+k}=$ $C_{p+2} h^{p+2} y^{p+2}(x)+O\left(h^{p+3}\right)$.

For our method, expanding (8) in Taylor series gives

$$
\begin{aligned}
\sum_{j=0}^{\infty} \frac{\left(\frac{1}{3} h\right)^{j}}{j !} y_{n}^{(j)}-y_{n}-\frac{1}{3} h y_{n}^{\prime}-\frac{28549}{10088640} h^{2} y_{n}^{\prime \prime} \\
-\sum_{j=0}^{\infty} \frac{h^{j+2}}{j !} y_{n}^{j+2}\left[\frac{275}{5184}\left(\frac{1}{3}\right)^{j}-\frac{5717}{120960}\left(\frac{2}{3}\right)^{j}+\frac{10621}{272160}(1)^{j}\right. \\
\left.-\frac{7703}{362880}\left(\frac{4}{3}\right)^{j}+\frac{403}{60480}\left(\frac{5}{3}\right)^{j}-\frac{199}{217728}(2)^{j}\right] \\
\sum_{j=0}^{\infty} \frac{\left(\frac{2}{3} h\right)^{j}}{j !} y_{n}^{(j)}-y_{n}-\frac{2}{3} h y_{n}^{\prime}-\frac{1027}{1707} h^{2} y_{n}^{\prime \prime}-
\end{aligned}
$$




$$
\begin{aligned}
& \sum_{j=0}^{\infty} \frac{h^{j+2}}{j !} y_{n}^{j+2}\left[\frac{194}{945}\left(\frac{1}{3}\right)^{j}-\frac{8}{81}\left(\frac{2}{3}\right)^{j}+\frac{788}{8505}(1)^{j}\right. \\
& \left.-\frac{97}{1890}\left(\frac{4}{3}\right)^{j}+\frac{46}{2835}\left(\frac{5}{3}\right)^{j}-\frac{19}{8505}(2)^{j}\right] \\
& \sum_{j=0}^{\infty} \frac{(h)^{j}}{j !} y_{n}^{(j)}-y_{n}-h y_{n}^{\prime}-\frac{25}{2688} h^{2} y_{n}^{\prime \prime}- \\
& \sum_{j=0}^{\infty} \frac{h^{j+2}}{j !} y_{n}^{j+2}\left[\frac{165}{448}\left(\frac{1}{3}\right)^{j}-\frac{267}{4480}\left(\frac{2}{3}\right)^{j}+\frac{5}{32}(1)^{j}\right. \\
& \left.-\frac{363}{4480}\left(\frac{4}{3}\right)^{j}+\frac{57}{2240}\left(\frac{5}{3}\right)^{j}-\frac{47}{13440}(2)^{j}\right] \\
& \sum_{j=0}^{\infty} \frac{\left(\frac{4}{3} h\right)^{j}}{j !} y_{n}^{(j)}-y_{n}-\frac{4}{3} h y_{n}^{\prime}-\frac{1088}{8505} h^{2} y_{n}^{\prime \prime}- \\
& \sum_{j=0}^{\infty} \frac{h^{j+2}}{j !} y_{n}^{j+2}\left[\frac{1504}{2835}\left(\frac{1}{3}\right)^{j}-\frac{8}{945}\left(\frac{2}{3}\right)^{j}+\frac{2624}{8505}(1)^{j}\right. \\
& \left.-\frac{8}{81}\left(\frac{4}{3}\right)^{j}+\frac{32}{945}\left(\frac{5}{3}\right)^{j}-\frac{8}{1701}(2)^{j}\right] \\
& \sum_{j=0}^{\infty} \frac{\left(\frac{5}{3} h\right)^{j}}{j !} y_{n}^{(j)}-y_{n}-\frac{5}{3} h y_{n}^{\prime}-\frac{35225}{217728} h^{2} y_{n}^{\prime \prime}- \\
& \sum_{j=0}^{\infty} \frac{h^{j+2}}{j !} y_{n}^{j+2}\left[\frac{8375}{12096}\left(\frac{1}{3}\right)^{j}+\frac{3125}{72576}\left(\frac{2}{3}\right)^{j}+\frac{25625}{54432}(1)^{j}\right. \\
& \left.-\frac{625}{24192}\left(\frac{4}{3}\right)^{j}+\frac{275}{5184}\left(\frac{5}{3}\right)^{j}-\frac{1375}{217728}(2)^{j}\right] \\
& \sum_{j=0}^{\infty} \frac{(2 h)^{j}}{j !} y_{n}^{(j)}-y_{n}+2 h y_{n}^{\prime}-\frac{41}{210} h^{2} y_{n}^{\prime \prime}- \\
& \sum_{j=0}^{\infty} \frac{h^{j+2}}{j !} y_{n}^{j+2}\left[\frac{6}{7}\left(\frac{1}{3}\right)^{j}+\frac{3}{35}\left(\frac{2}{3}\right)^{j}+\frac{68}{105}(1)^{j}-\frac{10}{70}\left(\frac{4}{3}\right)^{j}+\frac{6}{5}\left(\frac{5}{3}\right)^{j}\right]=0,
\end{aligned}
$$

comparing the coefficient efficient of $h$ gives $C_{0}=C_{1}=\ldots=C_{8}=0$ and

$$
C_{9}=\left[\begin{array}{llllll}
\frac{6031}{17856417600} & \frac{233}{279006525} & \frac{1}{765450} & \frac{496}{2796006525} & \frac{1621}{714256704} & \frac{1}{382725}
\end{array}\right]^{T}
$$


hence, our method is of order 7 .

\subsection{Zero Stability of the Method}

Definition 3. A block method is said to be zero stable if as $h \rightarrow 0$, the roots $r_{j}=1(1) k$ of the first characteristic polynomial $\rho(r)=0$ that is $\rho(r)=\operatorname{det}\left[\sum A^{(0)} R^{k-1}\right]=0$ satisfying $|R| \leq 1$, must have multiplicity equal to unity ( see Fatunla [8] for details)

For our method

$$
\begin{aligned}
\rho(R)= & {\left.\left[\begin{array}{llllll}
1 & 0 & 0 & 0 & 0 & 0 \\
0 & 1 & 0 & 0 & 0 & 0 \\
0 & 0 & 1 & 0 & 0 & 0 \\
0 & 0 & 0 & 1 & 0 & 0 \\
0 & 0 & 0 & 0 & 1 & 0 \\
0 & 0 & 0 & 0 & 0 & 1
\end{array}\right]-\left[\begin{array}{llllll}
0 & 0 & 0 & 0 & 0 & 1 \\
0 & 0 & 0 & 0 & 0 & 1 \\
0 & 0 & 0 & 0 & 0 & 1 \\
0 & 0 & 0 & 0 & 0 & 1 \\
0 & 0 & 0 & 0 & 0 & 1 \\
0 & 0 & 0 & 0 & 0 & 1
\end{array}\right]\right]=0, } \\
& \rho(R)=R^{5}(R-1)=0, \quad R=0,0,0,0,0,1 .
\end{aligned}
$$

Hence the method is zero stable.

\section{Stability Interval}

Definition 4. The block (8) is said to be absolutely stable within a given interval if for a given $h$, all roots $z_{s}$ of the characteristic polynomial $\pi(z, h)=$ $\rho(z)+h^{2} \sigma(z)=0$, satisfies $\left|z_{s}\right|<1, s=1,2, \ldots, n$. where $h=\lambda^{2} h^{2}$ and $\lambda=\frac{\partial f}{\partial y}$. We adopted the boundary locus method to determine the stability interval of our block.

Substituting $y^{\prime \prime}=-\lambda^{2} y$ into the block (8) and substituting $e^{i \theta}=\cos \theta+$ $i \sin \theta$, gives

$$
h^{-}=\frac{7437184000 \cos \theta-7437184000}{781 \cos \theta-12159}
$$

Hence the stability interval is given as $\theta \in\left[0,60^{\circ}\right]$

\section{Numerical Example}

Problem I. We consider the non-linear initial value problems

$$
y^{\prime \prime}=\frac{\left(y^{\prime}\right)^{2}}{2 y}-2 y, y\left(\frac{\pi}{6}\right)=\frac{1}{4}, y^{\prime}\left(\frac{\pi}{6}\right)=\frac{\sqrt{ } 3}{2}, h=0.05 .
$$




$$
\text { ExactSolution: } y(x)=(\sin x)^{2}
$$

This problem was solved by Awoyemi[6] and Awoyemi and Kayode [7], where they both proposed method implemented in predictor corrector method for stepsize $h=0.003125$. Awoyemi et al. [5] also solved this problem where a method implemented in block method was proposed for $h=0.003125$. Jator [10] and Adesanya et al. [2] also solved this problem where methods implemented in block method for stepsize $h=0.04913$ and 0.05 respectively were proposed. It was shown that the later method gave better approximation despite high value of $h$ used. We solved this problem using our method and compared our result with Adesanya et al. [2] and Jator [10] as shown in Table I.

Problem II. We consider a non linear initial value problem

$$
\begin{gathered}
y^{\prime \prime}-x\left(y^{\prime}\right)^{2}=0, y(0), y^{\prime}(0)=\frac{1}{2}, h=0.05 . \\
\text { ExactSolution: } y(x)=1+\frac{1}{2} \ln \left(\frac{2+x}{2-x}\right)
\end{gathered}
$$

This problem was solved by Jator [10] and Adesanya et al. [2], where a block method was proposed with stepsize 0.05. It was shown that the result of these authors are better than the existing methods. We solved this problem and compared our result with the results of these authors as shown in Table II.

Note: Error $=\mid$ Exact result-computed result $\mid$

$\mathrm{EAE}=$ Error in Adesanya et al [2]

$\mathrm{EJE}=$ Error in Jator [10]

Table 1 showing results for problem 1

\begin{tabular}{|l|l|l|l|l|l|}
\hline $\mathrm{X}$ & Exact solution & Computed Solution & Error in new method & EJE & EAE \\
\hline 1.10 & 0.77982668476381558 & 0.79982668475924446 & $4.5711(-12)$ & $2.8047(-10$ & $1.8811(-10)$ \\
\hline 1.20 & 0.87334365786466683 & 0.87334365785785987 & $4.7869(-12)$ & $2.7869(-10)$ & $2.4539(-10)$ \\
\hline 1.30 & 0.93197659748049533 & 0.93197659747586414 & $6.6311(-12)$ & $2.1490(-10)$ & $3.0306(-10)$ \\
\hline 1.40 & 0.97338799333525854 & 0.97338799333108894 & $4.6695(-12)$ & $5.4975(-10)$ & $3.5819(-10)$ \\
\hline 1.50 & 0.99592690375892334 & 0.99592690375564233 & $3.2810(-12)$ & $1.1545(-10)$ & $4.0838(-10)$ \\
\hline 1.60 & 0.99869477351706315 & 0.99869477351106584 & $5.9973(-12)$ & $4.4825(-10)$ & $4.5128(-10)$ \\
\hline 1.70 & 0.98158125637743932 & 0.98158125637252467 & $4.9146(-12)$ & $7.7969(-10)$ & $4.8473(-10)$ \\
\hline 1.80 & 0.94526861426358267 & 0.94526861425983988 & $3.7421(-12)$ & $1.1840(-09)$ & $5.0696(-10)$ \\
\hline 1.90 & 0.89120451762540223 & 0.89120451762278163 & $2.6205(-12)$ & $1.6318(-09)$ & $5.1697(-10)$ \\
\hline 2.00 & 0.82154433138674121 & 0.82154433137749383 & $9.2473(-12)$ & $2.0567(-09)$ & $5.1381(-10)$ \\
\hline
\end{tabular}

Table 1

Table 11 showing results for problem 11 


\begin{tabular}{|l|l|l|l|l|l|}
\hline $\mathrm{X}$ & Exact solution & Computed Solution & Error in new method & EJE & EAE \\
\hline 0.1 & 1.0500417292784914 & 1.0500417292784903 & $1.1102(-15)$ & $3.1624(-12)$ & $7.5028(-13)$ \\
\hline 0.2 & 1.1003353477310756 & 1.1003353477310696 & $5.9952(-15)$ & $1.5091(-11)$ & $9.7410(-12)$ \\
\hline 0.3 & 1.151140435936467 & 1.1511404359356441 & $2.5535(-14)$ & $4.5286(-11)$ & $3.7638(-11)$ \\
\hline 0.4 & 1.2027325540540823 & 1.2027325540540112 & $7.1054(-14)$ & $1.0808(-10)$ & $9.7765(-11)$ \\
\hline 0.5 & 1.2554128118829955 & 1.2554128118828796 & $1.1568(-13)$ & $1.7818(-10)$ & $2.0825(-10)$ \\
\hline 0.6 & 1.3095196042031119 & 1.3095196041202992 & $1.1990(-13)$ & $4.4424(-10)$ & $3.9604(-10)$ \\
\hline 0.7 & 1.3654437542713964 & 1.3654437542720822 & $6.8567(-13)$ & $7.4446(-10)$ & $7.0460(-10)$ \\
\hline 0.8 & 1.4236489301936022 & 1.4236489301970776 & $3.4754(-12)$ & $1.5009(-09)$ & $1.2095(-09)$ \\
\hline 0.9 & 1.484700278594052 & 1.4847002786062715 & $1.2219(-11)$ & $3.7579(-09)$ & $2.0511(-09)$ \\
\hline 1.0 & 1.5493061443340554 & 1.5493061443713383 & $3.7282(-11)$ & $4.7410(-09)$ & $3.5066(-09)$ \\
\hline
\end{tabular}

Table 2

\section{Conclusion}

We have proposed a direct method for the solution of second order initial value problems of ordinary differential equations. It has been shown that our new method is zero stable, consistent and convergent. The numerical examples have shown clearly that our method performs better in term of accuracy than the existing methods.

\section{References}

[1] S. Abbas, Derivation of a new block method for the numerical solution of first order IVPs, Intern. J. Comp. Math., 64, No. 4 (1997), 235-344

[2] A.O. Adesanya, M.R. Odekunle, A.O. Adeyeye, Continuous block hybridpredictor-corrector method for the solution of $y^{\prime \prime}=f\left(x, y, y^{\prime}\right)$, Intern. $J$. of Math. and Soft Computing, 3, No. 2, 35-42

[3] A.O. Adesanya, T.A. Anake, S.A. Bishop, J.A. Osilagun, Two steps block method for the solution of general second order initial value problems of ordinary differential equation, ASSET, 8, No. 1 (2009), 59-68.

[4] A.O. Adesanya, T.A. Anake, M.O. Udoh, Improved continuous method for direct solution of general second order ordinary differential equation, Journal of the Nigerian Association of Mathematical Physics, 13 (2008), 59-62

[5] D.O. Awoyemi, E.A. Adebile, A.O. Adesanya, T.A. Anake, Modified block method for the direct solution of second order ordinary differential equations, Intern. J. of Applied Mathematics and Computation, 3, No. 3 (2011), 181-188. 
[6] D.O. Awoyemi, A New Smith-order algorithm for general second order ordinary differential equation, Inter. J. Comp. Math., 77 (2001), 117-124.

[7] D.O. Awoyemi, S.J. Kayode, A maximal order collocation method for direct solution of initial value problems of general second order ordinary differential equation, In: Proceeding of the Conference Organized by the National Mathematical Centre, Abuja, Nigeria (2005).

[8] S.O. Fatunla, Parallel method for second order ordinary differential equation, In: Proceedings of the National Conference of Computational Mathematics, held at University of Benin, Nigeria (1992), 87 - 99.

[9] I.Yap Fudziah, H.K. Mohammed, Explicit and implicit 3-point block method for solving special second order ordinary differential equation directly, Intern. J. Math. Analysis, 3, No. 5 (2009), 239-252

[10] S.N. Jator, A sixth order linear multistep method for the direct solution of $y^{\prime \prime}=f\left(x, y, y^{\prime}\right)$, Intern. Journal of Pure and Applied Mathematics, 40, No. 1 (2007), 457-472.

[11] S.N. Jator, S. Li, A self starting linear multistep method for a direct solution of the general second order initial value problems, Intern. J. Comp. Math., 86, No. 5 (2009), 827-838.

[12] S.N. Jator, On a class of hybrid method for $y^{\prime \prime}=f\left(x, y, y^{\prime}\right)$, Intern. J. of Pure and Applied Mathematics, 59, No. 4 (2010), 381-395.

[13] S.J. Kayode, D.O. Awoyemi, A multiderivative collocation method for fifth order ordinary differential equation, J. of Mathematics and Statistics, 6 , No. 1 (2010), 60-63.

[14] B.T. Olabode, An accurate scheme by block method for the third ordinary differential equation, Pacific Journal of Science and Technology, 10, No. 1 (2009), http://www.okamaiuniversity.us/pjst.htm.

[15] Z. Omar, M. Suleiman, Parallel R-point implicit block method for solving higher order ordinary differential equation directly, Journal of ICT, $\mathbf{3}$, No. 1 (2003), 53-66.

[16] V.V. David Wend, Existence and uniqueness of solution of ordinary differential equation, Proceedings of the American Mathematical Society, 23, No. 1 (1969), 27-23. 